Ricardo Arraes de Al encar Ximenes 1

Celina Maria Turchi Martelli 2

Wayner Vieira de Souza 3

Tiago Maria Lapa 3

Maria de Fátima Militão de Albuquerque 4

Ana Lúcia S. Sampaio de Andrade 2

Otaliba Libânio de Morais Neto 2

Simonne de Al meida eSilva 2

Maria Luiza Carvalho de Lima 5

José Luiz Portugal 6

\section{Vigilância de doenças endêmicas em áreas urbanas: a interface entre mapas de setores censitários e indicadores de morbidade}

\author{
Surveillance of endemic diseases in urban areas: \\ the interface between census tract maps \\ and morbidity data
}

1 Departamento de Medicina Tropical, Universidade Federal de Pernambuco. Av. Moraes Rego s/no, Cidade Universitária, Recife, PE, 50670-420, Brasil.

rximenes@el ogica.com.br

2 Instituto de Patologia

Tropical e Saúde Pública, Universidade Federal de Goiás. Rua Delenda Resende de Melo, s/no, Setor

Universitário, Goiânia, GO 74605-050, Brasil.

3 Núcleo de Informação Científica, Centro de

Pesquisas Aggeu Magalhães, Fundação Oswaldo Cruz.

Av. Moraes Rego s/no,

Cidade Universitária,

Recife, PE, 50670-420, Brasil.

4 Núcleo de Estudos em

Saúde Coletiva, Centro de

Pesquisas Aggeu Magalhães,

Fundação Oswaldo Cruz.

Av. Moraes Rego s/no,

Cidade Universitária,

Recife, PE, 50670-420, Brasil.

5 Coordenação de Pós-

Graduação, Universidade de Pernambuco.

Rua Arnóbio Marques 310,

Campus Universitário,

Santo Amaro, Recife, PE

50100-420, Brasil.

6 Departamento de

Engenharia Cartográfica,

Universidade Federal

de Pernambuco.

Av. Moraes Rego s/no,

Cidade Universitária,

Recife, PE, 50670-420, Brasil.
Abstract In this article we discuss the methodological issues associated with the creation of a surveillance system for endemic di seases in urban areas based on analysis of populations at risk and on spatially referenced epidemiological indicators. We comment on the system's basic requirements, selection criteria for soci oeconomic variables, and methodological steps to combine these variables so as to construct a census-based deprivation index. Weal so present the ways we solved some operational problems related to generation of digitized census tracts maps and linkage of morbidity data from different sources. This approach, spatial organization into account in surveillance of endemic diseases, exemplified here by tuberculosis and leprosy, allows for the interaction of several official data sets from census and heal th services in order to geographically discriminate inner-city risk strata. Criteria for constructing these risk strata were consi dered a useful tool for health planning and management activities for the control of endemic diseases in cities.

Key words Epidemiologic Surveillance; Leprosy; Tuberculosi s; Spatial Distribution; Health Status Indicators

Resumo Nesteartigo, discute-se o eixo metodológi co utilizado na construção de model o de vigilância de endemias em áreas urbanas, orientado por uma análise de si tuações de risco e por indicadores epi demiológi cos espaciais. São apresentadas as premi ssas bási cas do model o, os critéri os de sel eção de variávei s sóci o-econômi cas e as etapas metodol ógicas necessárias na construção do indicador sintético de risco. Comenta-se, também, como al gumas questões operacionais relativas à construção de mapas di gitais de setores censitários e vinculação de ban cos de dados foram equacionadas. Essa abordagem, incorporando o componente da organização do espaço na vigilância de doenças endêmicas, ten do como exemplo a hanseníase e a tuberculose, privilegia o uso integrado de sistemas de informação já existentes, na perspectiva de estratificar áreas urbanas diferenciadas que permite di scriminar riscos desiguais para ocorrência de endemias. Essa é uma ferramenta para o planejamento e o gerenciamento das ações voltadas para o controle das endemias nas cidades.

Palavras-chave Vigilância Epidemiológica; Hanseníase; Tuberculose; Distribuição Espacial; Indicadores de Saúde 


\section{Introdução}

No Brasil, pouco se conhece sobre a distribuição espacial dos eventos mórbidos, particularmente do padrão urbano das doenças endêmicas (Sabroza et al., 1992; Albuquerque, 1993). A expansão de endemias rurais para regiões urbanas, imputada aos deslocamentos populacionais, ocorreu de forma predominante nas décadas de 60 e 70. Atualmente, a urbanização da população e o fenômeno de periferização das metrópoles constituem-se no cenário demográfico dominante, tornando ainda mais complexo o controle da transmissão intra-urbana de algumas endemias (Andrade, 1990; Martelli et al., 1995; Barcelos \& Bastos, 1996). Essa rápida e desordenada periferização das populações vem criando bolsões de carência social e de exclusão de atendimento em saúde, exigindo novas estratégias de controle das endemias.

A construção de um sistema de vigilância da saúde, orientado por um modelo de análise de situações de risco em substituição ao modelo de risco individual, utiliza o espaço como referência e tem potencial mente um maior poder explicativo por expressar diferentes acessos aos bens e serviços de infra-estrutura urbana (Mendes et al., 1994; Santos, 1996). Este enfoque evidencia a desigualdade existente no interior das cidades, possibilitando o planejamento de intervenções e monitoramentos seletivos conforme as reais necessidades de pequenas áreas.

No presente artigo, discute-se o eixo metodológico na construção de sistema de vigilância de endemias em áreas urbanas, orientado por indicadores epidemiológicos espaciais, que vem sendo implementado nos Municípios de Goiânia, capital do Estado de Goiás, e de Olinda, Estado de Pernambuco. O enfoque da presente discussão é estabelecer quais as premissas utilizadas na construção do modelo de vigilância da saúde. Nesta perspectiva, são apresentados os critérios de seleção de váriáveis sócio-econômicas, as etapas metodológicas na construção do indicador composto e a estratificação espacial das situações de risco, possibilitando o planejamento de intervenções e monitoramentos seletivos. Os dados de hanseníase e tuberculose são utilizados como exemplo por serem eventos de notificação compulsória com sistemas de informações estáveis e bem estruturados. Comenta-se, também, como algumas questões operacionais relativas à implementação de mapas urbanos digitais em saúde foram equacionadas.
Pressupostos do modelo de vigilância da saúde

Três pressupostos básicos orientaram a concepção desta proposta: a) o conceito de vigilância da saúde, que transcende a clássica noção de vigilância no nível do indivíduo doente ou de seus comunicantes, para priorizar a vigilância do espaço/ população de ocorrência da doença. Nesse contexto, um indicador composto que sintetize as condições de vida foi construído para identificar áreas críticas intra-urbanas; b) a identificação de áreas de diferentes níveis de endemicidade sinalizadas pelos indicadores de morbidade, as quais, superpostas aos bolsões sociais de carência, localizam espacialmente os grupos sociais para intervenções e monitoramento seletivos de múltiplos agravos e c) a crescente transferência de responsabilidade do planejamento e execução das atividades de controle de endemias para os municípios, correspondendo à política oficial do Sistema Único de Saúde (SUS) de repasse de financiamento, vinculado à análise epidemiológica local (Norma Operacional Básica).

O desenvolvimento metodológico do modelo de vigilância epidemiológica proposto encontra-se diagramado na Figura 1. As regiões, distritos urbanos e sanitários, representam os limites geográficos de administração municipal, macroáreas heterogêneas do ponto de vista populacional e sócio-econômico. Já os setores censitários representam a unidade espacial mais desagregada para a qual se dispõe de dados, periodicamente atualizados, referentes à estrutura demográfica e características sociais da população.

\section{Unidade espacial de análise - limites geográficos}

Estabeleceram-se os setores censitários como a unidade de menor nível de agregação espacial. Para o Município de Goiânia, a contagem populacional de 1996 (IBGE, 1996) alterou os limites geográficos de cerca de $25 \%$ dos setores censitários (divididos, reagrupados e expandidos), para adequar as unidades espaciais à atual estrutura populacional e territorial da cidade (Figura 2). Esses novos limites geográficos foram utilizados na construção de mapas censitários digitais, por retratarem a presente configuração espacial da cidade e serem compatíveis com as unidades espaciais de gerência municipais. Para o Município de Olinda, por se tratar de área altamente adensada e de população relativamente estável na última década, utilizou-se a estrutura espacial do censo de 1991 (IBGE, 1991). 
Figura 1

Modelo teórico de vigilância epidemiológica georreferenciada.

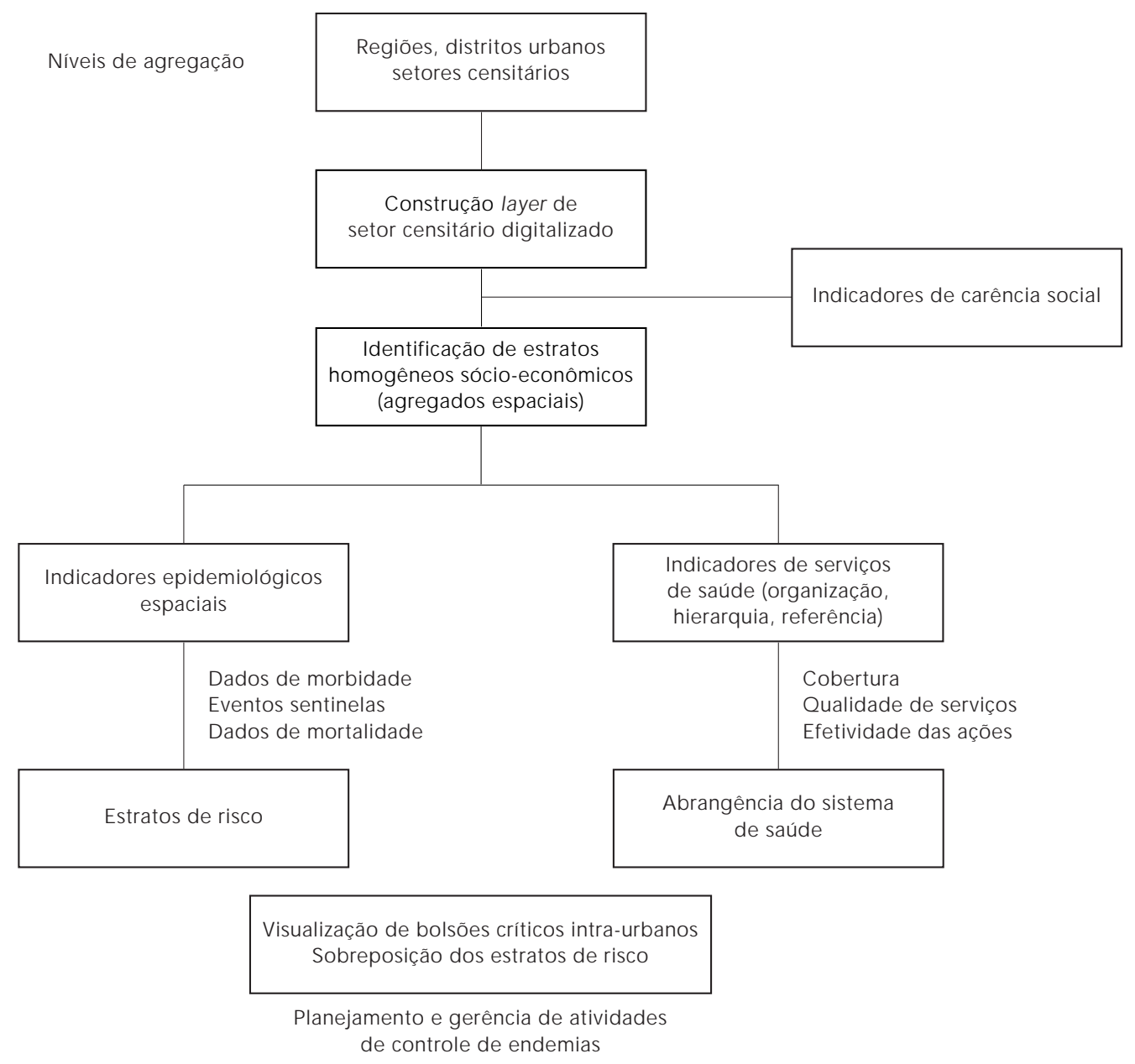

Figura 2

Compatibilização da estrutura de setores censitários do Censo (IBGE - 1991) com o mini-Censo de 1996 para o Município de Goiânia, Estado de Goiás.

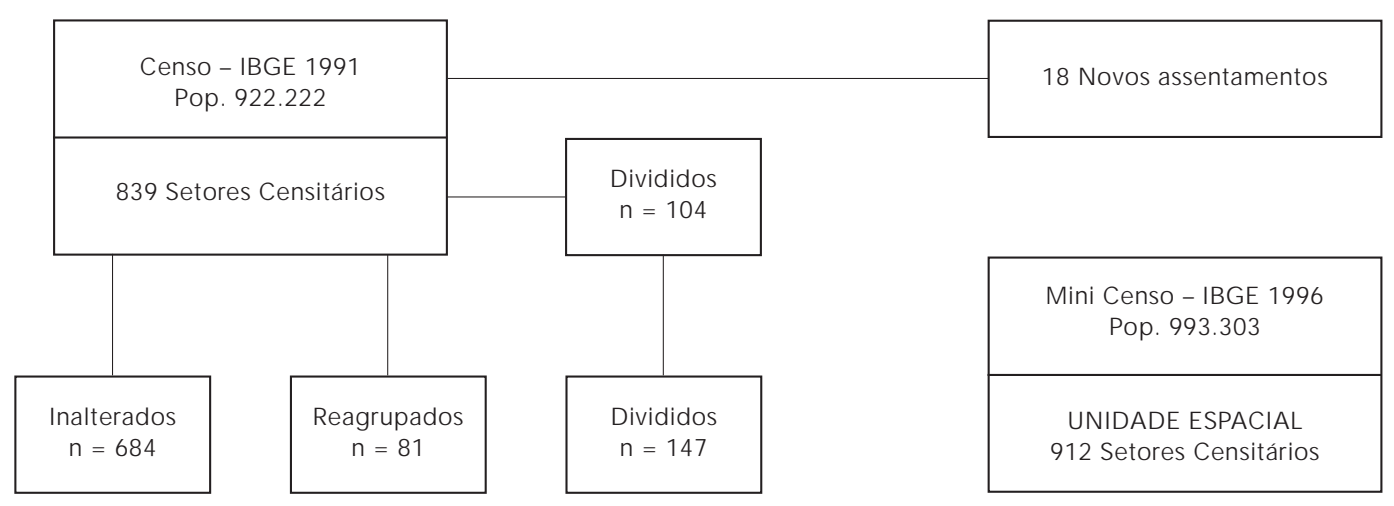

Novos assentamentos Mobilidade populacional Compatibilização dos Setores com distritos administrativos 
Construção da base territorial digitalizada

O Município de Goiânia está localizado a 49。 $00^{\prime}$ - 49o 45' W, 160 30' - 170 00' S, com 989.285 habitantes, área de $789,87 \mathrm{~km}^{2}$, densidade demográfica de 1252,7 hab./ km². O Mapa Urbano Básico Digital de Goiânia (MUBDG), concluído em 1996, foi fornecido pela Comdata, que é a instituição municipal responsável pela construção da malha digital da cidade. O MUBDG encontra-se em formato CAD e contém os limites geográficos em coordenadas UTM (Projeção universal transversa de Mercator) para distritos urbanos, bairros, quadras e lotes. Esse mapa digital urbano foi utilizado como layer básico na digitalização dos 912 setores censitários. Para a delimitação de tais setores foram utilizados os mapas de campo e memorial descritivo (IBGE, 1996). A média de habitantes por setor censitário é de aproximadamente 980 habitantes.

O Município de Olinda está localizado a 340 $49^{\prime}$ - 340 54' W, 70 57' - 80 02' S, com uma população de 341.387 habitantes, área aproximada de $40 \mathrm{~km}^{2}$, com densidade demográfica de $9062 \mathrm{hab} . / \mathrm{km}^{2}$. A média de habitantes por setor censitário em Olinda é de 1.405 habitantes. O Mapa Urbano Básico Digital de Olinda, contendo basicamente os limites do Município e arruamento, foi fornecido pela Fundação de Desenvolvimento da Região Metropolitana (Fidem), instituição responsável pelo projeto Unibase, que objetiva a construção de mapas digitais dos municípios da região metropolitana do Recife. Com base nesse mapa, digitalizou-se o layer de 243 setores, a partir dos seus descritores de limites, de acordo com o censo demográfico de 1991. Esse tipo de mapa de setores censitários reflete a distribuição da população, permitindo visual izar os maiores adensamentos populacionais, configurando um mapa de base populacional (Figura 3).

Arquitetura e modelagem

de bancos de dados

Censo demográfico - Os arquivos originais provenientes do censo de 1991, em formato DAT, foram convertidos para o formato DBF. Este banco de dados contém dados de população, bem como caracterizações sócio-econômica e dos domicílios relativas a cada setor censitário. Foram também utilizados os dados de população da contagem populacional para o Município de Goiânia (IBGE, 1996). Nesse sentido, necessitou-se de uma adequação das variáveis oriundas do censo de 1991 à atual estrutura espacial dos setores censitários definidos em 1996, conforme mostra a Figura 2. O linkage direto foi possível para os setores censitários que permaneceram inalterados (75\%) entre os dois censos. Para os restantes $25 \%$, foi realizada uma adequação das variáveis do censo (1991), levando-se em conta a atual população e a distribuição proporcional das variáveis sócio-econômicas nessa nova estrutura espacial.

Banco de dados de morbidade - Selecionaram-se indicadores epidemiológicos já estabelecidos pelos programas de controle como estratégia para caracterizar áreas de maior endemicidade. Os dados do Sinan para hanseníase e tuberculose foram utilizados para o cálculo dos coeficientes anuais de deteç̧ão de casos por setor censitário no período de 1991-1996. Em razão das características de análise epidemiológica em pequenas áreas e considerandose que hanseníase e tuberculose são doenças de evolução crônica, construíram-se coeficientes médios para o período, obtendo-se indicadores de morbidade mais estáveis e mais representativos da distribuição espacial dessas endemias.

Além disso, calcularam-se coeficientes por faixa etária, uma vez que é reconhecido que casos de tuberculose e hanseníase em menores de 15 anos podem sinalizar áreas de transmissão recente (MS, 1996). Outro tipo de abordagem foi a utilização de eventos-sentinela, ou seja, ocorrência de eventos de maior potencial de gravidade em uma microárea, possibilitando um esquadrinhamento do espaço urbano para intervenções seletivas de controle das endemias.

Esse tipo de análise de informações referentes a desagregados espaciais intra-urbanos torna visíveis as desigualdades dos grupos populacionais e é um avanço em relação às abordagens tradicionais por grandes áreas, que não possibilitam o uso da informação de morbidade para planejamento e gerenciamento das ações voltadas para o controle das endemias.

Vale ressaltar que a variável que localiza os bancos de dados de morbidade é, em geral, o endereço de residência. A vinculação dessas informações na perspectiva territorial implica agregação dos dados de acordo com a unidade espacial de estudo. Nessa proposta, todos os endereços referentes às notificações foram convertidos em seus respectivos setores censitários, para se obter um link com os mapas digitais. Esses procedimento de conversão de dados de localização foi trabal hoso e consumiu grande parte do tempo das equipes envolvidas, 
por causa dos endereços incompletos dos arquivos de morbidade e também porque as características de arruamento não permitem realizar esta etapa de forma automatizada. Pesquisas em múltiplas fontes, correios, lista telefônica, visitas domiciliares etc. foram utilizadas para viabilizar essa transformação. No entanto, considerando-se a série histórica de hanseníase e tuberculose de 1991-1996 para o Município de Olinda, foi possível, pelo endereço de notificação, georreferenciar, nos setores censitários, cerca de $80 \%$ dos casos em todos os anos (Tabela 1). Partindo-se do pressuposto de que os endereços incompletos, portanto difíceis de serem localizados, pertencem a áreas carentes, poderíamos estar incorrendo em um erro de subestimar os casos em bolsões urbanos de pobreza.

\section{Construção do indicador composto de situação de risco e definição de estratos}

Um indicador composto de carência social foi construído utilizando-se as seguintes variáveis do censo (IBGE, 1991): renda, educação, abastecimento de água, habitantes por dormitório, habitação subnormal e densidade demográfica. Essas variáveis foram selecionadas por refletirem classicamente condição social e qualidade de infra-estrutura urbana e por representarem informações que teórica e empiricamente estão relacionadas com as endemias em estudo (Albuquerque, 1993; Albuquerque \& Morais, 1997).

Cada uma das variáveis selecionadas foi transformada em valores zero a um, onde um indica as maiores carências e zero, as menores, conforme metodologia adaptada da classificação dos municípios brasileiros e do Índice de Desenvolvimento Humano (Unicef, 1994). Assim, cada setor censitário recebeu uma pontuação que corresponde à média aritmética dos escores. Por meio do ordenamento e da divisão em quartis ou quintis, foi possível discriminar estratos urbanos homogêneos enquanto categoria de risco, expressando de forma sintética as condições de vida. A Figura 3 mostra como os bairros e setores de planejamento, que correspondem às divisões geopolíticas, abrangem áreas heterogêneas do ponto de vista das condições de vida, e, conseqüentemente, das necessidades de saúde.

Para validar e mesmo visualizar como as variáveis sócio-econômicas escolhidas distribuem-se nos diferentes estratos de risco, estabelecemos a correlação entre os indicadores
Figura 3

Mapa do Município de Olinda:

a) Distribuição espacial dos setores censitários com respectivas densidades populacionais; b) Mapa de bairros, distritos e áreas programáticas;

c) Superposição de estratos de risco com áreas programáticas.

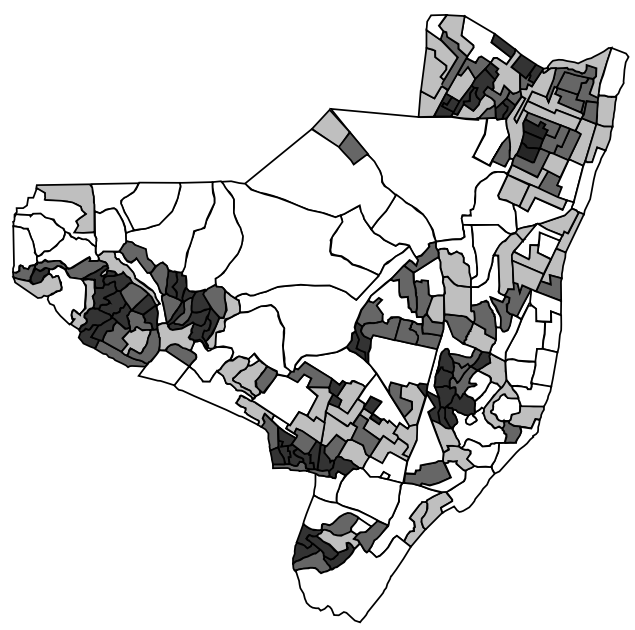

Figura $3 a$

$\mathrm{Hab} / \mathrm{km}^{2}$

$360.5-7877.9$

$7877.9-12734.6$

$12734.6-18944.3$

$18944.3-52806.9$

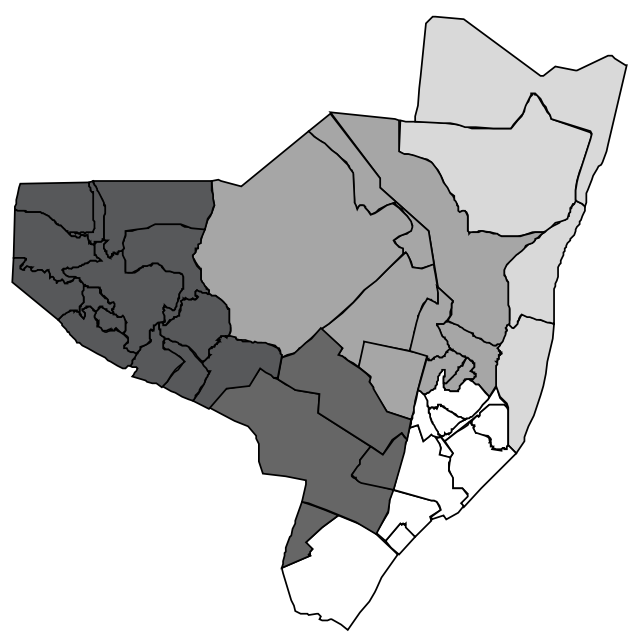

Figura 3b

Bairros

Distrito 1

Área I

Área II

Distrito 2

Área III

Área IV

Área V

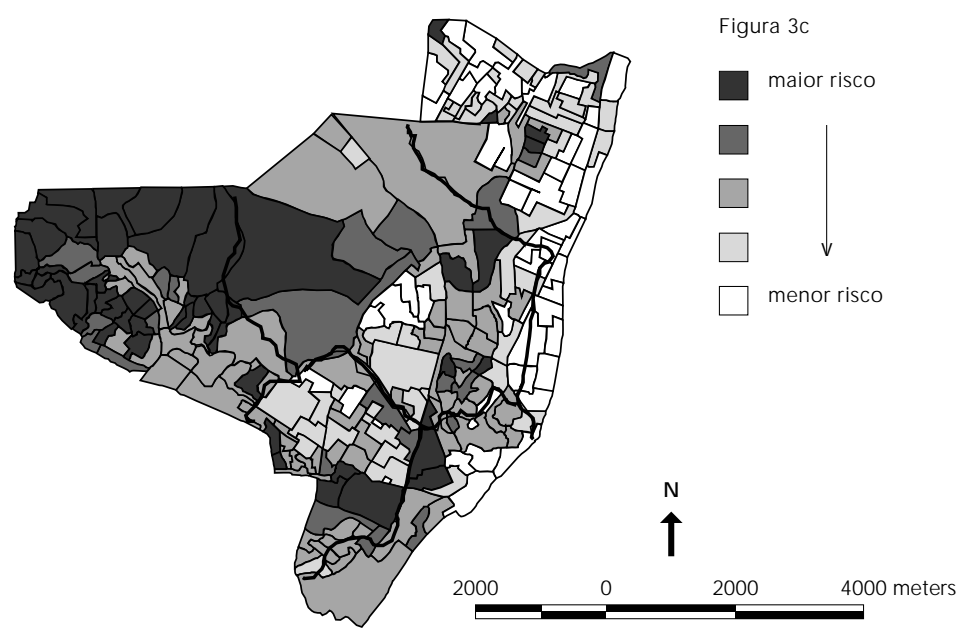


Tabela 1

$\mathrm{N}$ úmero de casos de hanseníase notificados e respectivos percentuais de localização nos setores censitários para o Município de Olinda, no período 1991-1996.

\begin{tabular}{lcc}
\hline Ano & Casos & Referenciados (\%) \\
\hline 1991 & 240 & 85,0 \\
1992 & 230 & 81,7 \\
1993 & 268 & 82,8 \\
1994 & 256 & 86,4 \\
1995 & 329 & 91,2 \\
1996 & 352 & 84,4 \\
Total & 1.675 & 85,5 \\
\hline
\end{tabular}

padronizados e os estratos construí dos através do ordenamento do indicador sintético. No exemplo apresentado para o Município de Goiânia, as caixas representam $75 \%$ da distribuição e a linha central, a mediana dos valores, como mostram os gráficos de caixa (Figura 4) (Erickson \& Nokanchuk, 1995). Neste tipo de análise exploratória, podemos observar o padrão de distribuição de cada variável sócio-econômica de acordo com os estratos de risco definidos por quartis. Há uma correlação nítida entre as variáveis selecionadas e os estratos de risco estabelecidos, sugerindo validade e coerência nesta abordagem. Ressaltamos que outras variáveis sócio-econômicas e epidemiológicas poderiam ser selecionadas de acordo com as características das áreas urbanas e da endemia em estudo.

Vantagens e limitações da metodologia utilizada

A utilização dos setores censitários, como unidade espacial de análise no desenvolvimento do modelo em estudo, apresenta a vantagem de representar o nível mais desagregado de dados populacionais e sócio-econômicos coletados de forma sistemática, periódica e padronizada com abrangência nacional. Além disso, é possível analisar os dados em diferentes níveis de agregação, de acordo com o detalhamento espacial desejado pelas instâncias de planejamento e gerência. Esse tipo de análise, ao identificar bolsões de carência e de ocorrência dos agravos em mi croáreas de forma simultânea e interativa, permite implementar ações sel etivas e específicas de controle das endemias.
A seleção de informações do censo por critérios não estatísticos, como a utilizada para formação de escores, pode ser uma das limitações da metodologia empregada, uma vez que cada variável apresenta o mesmo peso no escore final. Porém, esta lógica simples de sintetizar a situação sócio-econômica é justificável por ser de fácil manuseio e compreensão para os serviços de saúde. Outras técnicas, como, por exemplo, a análise fatorial/ componentes principais, vêm sendo empregadas academicamente para selecionar variáveis do censo, mediante critérios estatísticos, para composição do indicador sintético de risco (Rodriguez, 1996). Entretanto, vale ressaltar que cada endemia apresenta particularidades em sua forma de transmissão e propagação, e esse conhecimento empírico deve ser considerado na escolha do conjunto de fatores a serem incorporados na construção do indicador de risco.

Uma outra questão refere-se a um certo grau de arbitrariedade para estabelecer pontos de corte na discriminação dos estratos de risco. Em contrapartida, a abordagem empregada de ordenamento e divisão de estratos pode ser adequada ao conhecimento prévio sobre o território de estudo.

\section{Perspectivas}

A recente disponibilidade de mapas digitais para algumas cidades representa, sem dúvida, um avanço para o planejamento integrado de diferentes setores. Nesse sentido, o interesse das secretarias municipais de saúde no uso de Sistema de Informação Geográfica (SIG) para esquadrinhamento da área urbana tem sido notável (Brêtas \& Bessa, 1996; Nobre et al., 1996; Soares Filho \& Sawyer, 1996; Fator GIS, 1997), embora o geoprocessamento em saúde venha sendo incorporado, basicamente, como ferramenta de visualização de eventos de saúde em mapas, sem definição de uma proposta epidemiológica conceitual de vigilância do espaço. Neste artigo, o delineamento do modelo de vigilância proposto privilegia a compreensão do processo de produção das doenças em razão da ocupação do espaço urbano, com perspectiva de integração e compatibilização de bancos de dados existentes e análise interativa de dados de saúde e sócio-demográficos. Possibilita, também, como mostramos, articular dados disponíveis de diversos setores por meio de análise de dados de caráter exploratório, trazendo essas informações sumarizadas em índices de carência social sobrepostos em áreas de maior ocorrência dos eventos, para o nível local onde 


\section{Figura 4}

Correlação entre indicadores sócio-econômicos padronizados e o Índice de Qualidade de Vida (IQV) nos setores censitários de Goiânia-GO.
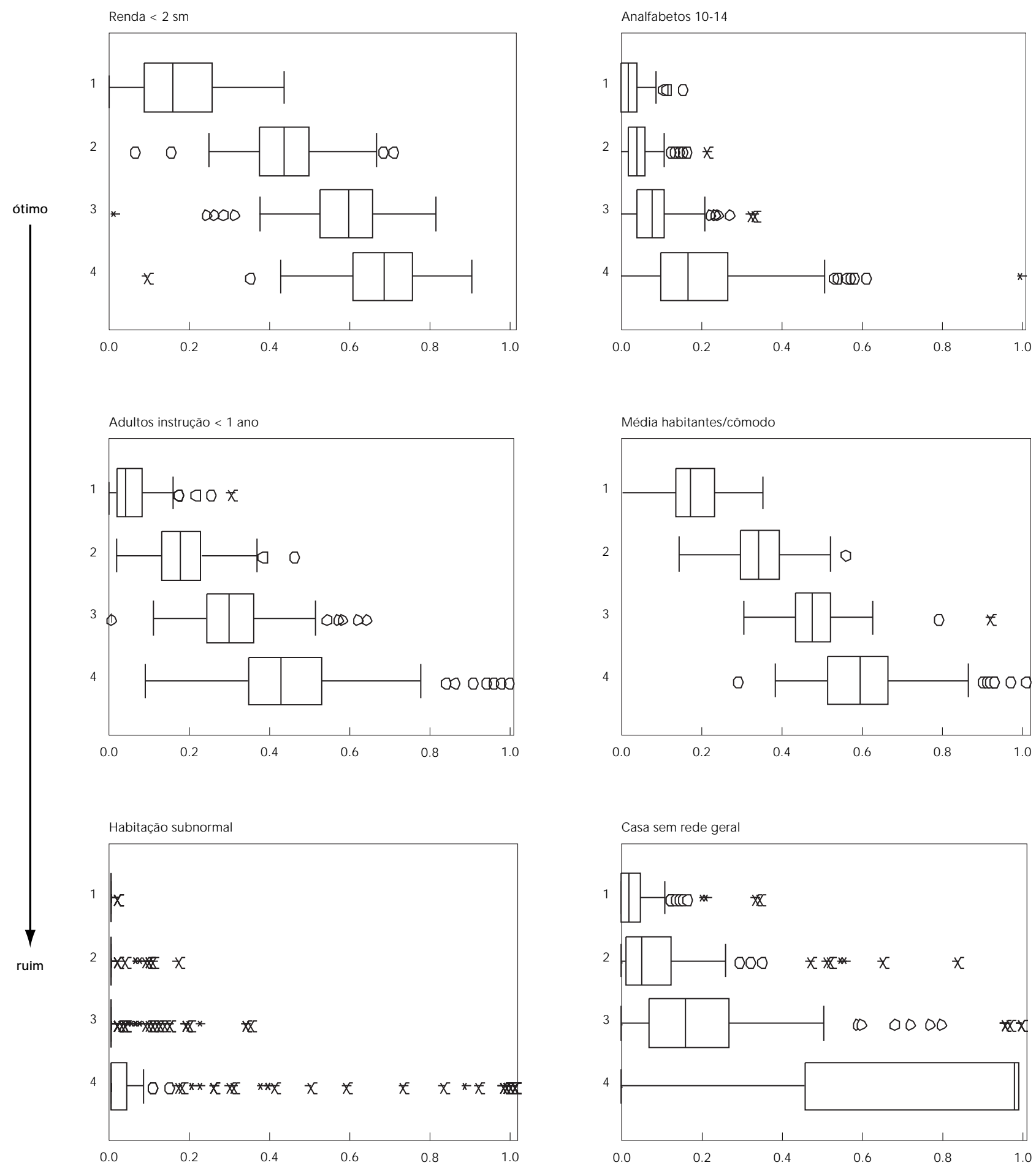

menor carência

maior carência 
as ações devem se dar. Esse tipo de aproximação na abordagem dos eventos de saúde em área urbana antecede a aplicação de modelos mais complexos de análise espacial de dados com hipóteses previamente definidas, para testar tendências, regularidade e existência de conglomerados, que sejam estatisticamente significantes, levando em conta o seu padrão de dis-

\section{Agradecimentos}

Agradecemos a Proade/ CENEPI/Fundação Nacional de Saúde pelo apoio financeiro ao projeto de Vigilância de Doenças Endêmicas em Áreas Urbanas - Construção de Indicadores Operacionais e Epidemiológicos Georreferenciados. Agradecemos também a Flávio Yuaça da COMDATA de Goiânia, Nhyro Gonçalves Laranja Filho da Coordenação de Geociências da Fundação IBGE-Goiás e ao Professor Francisco Jaime Bezerra Mendonça do Departamento de Engenharia Cartográfica da UFPE pela assessoria e apoio na digitalização dos mapas. Agradecemos ainda aos Técnicos da Fundação Nacional de Saúde - CR GO/PE e Secretarias Municipais e Estaduais de Saúde pela participação na fase de elaboração da proposta de trabaIho e a Giselle Gouveia, do Núcleo de Informação Científica do CPqAM, pela colaboração na espacialização dos casos. tribuição espacial (Carvalho et al., 1996; Gatrell \& Bailey, 1996). Em conclusão, apresentamos um modelo de vigilância à saúde, que transcende o monitoramento de indivíduos doentes, que estimula a articulação intersetorial, que se enquadra nas diretrizes do SUS, possibilitando, desta forma, estabelecer uma lógica para intervenções que sejam diferenciadas e seletivas.

\section{Referências}

ALBUQUERQUE, M. F. M., 1993. Urbanização, favelas e endemias: a produção da filariose no Recife, Brasil. Cadernos deSaúde Pública, 9:487-497.

ALBU QUERQUE, M. F. M. \& MORAIS, H. M. M., 1997. Descentralization of endemic disease control: an intervention model for combating bancroftian filariasis. Revista Panamericana de Salud Pública/Pan American Journal of Public Health, 1:155163.

ANDRADE, V. L. G., 1990. Características epidemiológi cas da Hanseníase em Área Urbana: Município de São Gonçalo. Dissertação de mestrado, Rio de Janeiro: Escola Nacional de Saúde Pública, Fundação Oswaldo Cruz.

BARCELOS, C. \& BASTOS, F. I., 1996. Redes sociais e difusão da AIDS no Brasil. Boletín de la Oficina Sanitaria Panamericana, 121:11-24.

BRÊTAS, G. \& BESSA, R., 1996. Um sistema geográfico de informações para o controle da malária na Amazônia. Informe Epidemiológico do SUS, 5:7381.

CARVALHO, M. S.; CRUZ, O. G. \& NOBRE, F. F., 1996. Spatial partioning using multivariate cluster analysis and a contiguity al gorithm. Statistics in Medicine, 15:1885-1894. 
ERICKSON, B. H. \& NOKANCHUK, T. A., 1995. Understanding Data: an Introduction to Exploratory and Confirmatory Data Analysis for Students in Social Science. 2nd ed. London: Open University Press.

FATOR GIS, 1997. Saúde para as cidades: o geoprocessamento na Saúde Pública. Fator GIS, 5:28-35.

GATRELL, A. C. \& BAILEY, T. C., 1996. Interactive spatial data analysis in medical geography. Social Science and Medicine, 42:843-855.

IBGE (Instituto Brasileiro de Geografia e Estatística), 1991. Censo Demográfico 1991. Rio de Janeiro: IBGE.

IBGE (Instituto Brasileiro de Geografia e Estatística), 1996. Contagem Populacional 1996. Rio de Janeiro: IBGE.

MARTELLI, C. M. T.; MORAES NETO, O. L.; ANDRADE, A. L. S. S.; SILVA, S. A.; SILVA, I. M. \& ZICKER, F., 1995. Spatial patterns of leprosy in an urban area in Central Brazil. Bulletin of theWorld Health Organization, 73:315-319.

MENDES, E. V.; TEIXEIRA, C. F.; ARAUJO, E. C. \& CARDOSO, M. R. L., 1994. Distritos sanitários: conceitos - chave. In: O Processo Social de Mudança das Práticas Sanitárias do Sistema Único de Saúde (E. V. Mendes, org.), pp. 159-185, São Paulo: Hucitec/ Rio de Janeiro: Abrasco.

MS (Ministério da Saúde), 1996. Instrumento de avaliação do Programa Nacional deControlee Eliminação da Hanseníase. Brasília: Ministério da Saúde. (mimeo.)
NOBRE, F. F.; BRAGA, A. L; PINHEIRO, R. S. \& LOPES, J. S., 1996. SIGEPE: um sistema básico de informação geográfica para o apoio a vigilância epidemiológica. Informe Epidemiológico do SUS, 5:59-72.

RODRIGUEZ, P. D., 1996. Variações Espaciais de Fatores de Risco em Saúde em Áreas Geográficas Pe quenas. Dissertação de Mestrado, Rio de Janeiro: Engenharia Biomédica, Universidade Federal do Rio de Janeiro.

SABROZA, P. C., TOLEDO, L. M. \& OSANAI, C. H., 1992. Organização do espaço e os processos endêmicos epidêmicos. In: Saúde, Ambiente e Desenvolvimento, Vol. II, (M. C. Leal, org.), pp. 52-77, São Paulo: Hucitec/Rio de Janeiro: Abrasco.

SANTOS, M., 1996. A Natureza do Espaço. Técnica e Tempo. Razão e Emoção. São Paulo: Hucitec.

SOARES FILHO, B. S. \& SAWYER, D., 1996. Correlatos ambientais da distribuição espacial da malária em municípios endêmicos no norte do estado do Mato Grosso. Informe Epidemiológico do SUS, 5: 47-57.

UNICEF (Fundo das Nações Unidas para a Infância), 1994. Municípios Brasileiros: Crianças e suas Condições de Sobrevivência. Brasília: Fundação Instituto Brasileiro de Geografia e Estatística. 